\title{
Rapid and sensitive precipitin test for the diagnosis of farmer's lung using immunoosmophoresis ${ }^{1}$
}

\author{
J. E. JAMESON
}

From the Public Health Laboratory, Brighton

SYNOPSIS An agar gel precipitin test, using osmophoresis, has been devised for use with weakly reacting sera. The test was applied to various categories of sera, including sera from cases of farmer's lung which had reacted with a conventional immuno-diffusion test, and others which had not.

All the conventionally sero-positive sera reacted positively in the new test as did nine of 16 cases in the sero-negative group. Concentration of sera in the latter group resulted in 11 positive tests out of a possible 12. Evidence was obtained of qualitative differences in the sera of these two groups.

Unexpected reactivity with sera from cases of farmer's lung was found in a dialysate from a broth culture of $T$. polyspora. No reactions with this dialysate were obtained with the sera of any patients who did not have symptoms of farmer's lung.

When an electric current is passed through a negatively charged gel such as agar, water molecules move from the anode towards the cathode; this phenomenon of endosmosis slows the migration of rapidly moving negatively charged bodies and may cause slow moving ones to reverse their direction of flow. Thus when $\gamma$-globulins are electrophoresed in such a gel at a $p \mathrm{H}$ between $7 \cdot 2$ and 9.0 they migrate towards the cathode. Since the flow of the soluble antigens of Thermopolyspora polyspora (Pepys, Jenkins, Festenstein, Gregory, Lacey, and Skinner, 1963) under similar conditions is mainly from cathode to anode, this method may be used to force these antigens and globulins to converge on each other. In conventional immunodiffusion techniques losses occur through dilution and by migration of the reactants in unwanted directions. This leads to a loss of sensitivity of such degree that sera of low titre may fail to react visibly.

Culliford (1964) made use of immunoosmophoresis to detect soluble antigens in blood stains and other forensic specimens. Adequately titred test sera were available for his purpose. When testing for precipitins which may be present in low titre sera, one inch slotted serum origins, as shown in Fig. 1, were found more suitable than the small circular wells used by Culliford. The higher sensitivity thus obtained enabled a wider initial separation of the reactants, with consequent improved' resolution of the pre-

${ }^{1} A$ report to the working party on farmer's lung of the Public Health Laboratory Service.

Received for publication 24 October 1967. cipitin bands. A strip of Whatman 3 MM paper, soaked in antigen and laid transversely on the surface of the agar, distorts ionic flow during electrophoresis less than an antigen-containing slot in the corresponding position. Even though antigen in a slot may have the same ionic strength as the surrounding agar, it usually empties from the slot during osmophoresis. Precipitin bands may develop in the agar lying beneath a paper strip, and also on its cathodic side. The lower holding capacity of the paper is compensated by the large size of the strip and by the use of concentrated antigen (Fig. 2, a-f).

\section{MATERIALS AND METHODS}

BUfFER SOLUTION This was a veronal buffer (Pepys et al., 1963) prepared as follows: sodium barbitone $82.48 \mathrm{~g}$, diethyl barbituric acid $14.736 \mathrm{~g}, 1 \%$ Thiomersal $40 \mathrm{ml}$, deionized water to 4 litres. The $p \mathrm{H}$, by measurement, was 8.6 . This is referred to as $N / 1$ buffer. $N / 2$ buffer was used

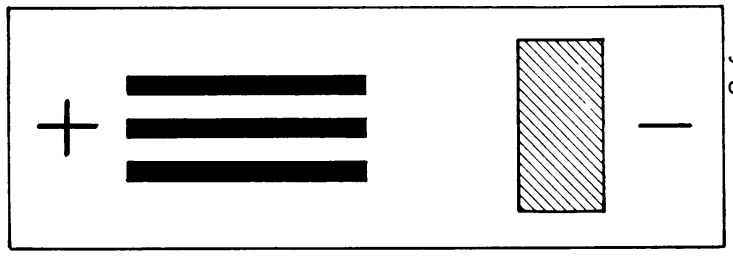

FIG. 1. Agar shown on a glass slide, 3 in. $\times 1$ in. before osmophoresis. Black areas are serum slots $\left(1\right.$ in. $\times \frac{1}{16}$ in. $)$ and the shaded area is antigen strip $\left(\frac{3}{4}\right.$ in. $\times \frac{3}{8}$ in. $)$. 
in the electrophoresis tank and N/2.5 buffer in agar slides.

ANTIGEN A concentrated broth culture extract of $T$. polyspora was prepared as follows: $T$. polyspora was floated on the surface of $1 \%$ glucose broth in $100 \mathrm{ml}$ amounts in wool-plugged $20 \mathrm{oz}$ medical flats, and incubated at $52^{\circ} \mathrm{C}$ for approximately six weeks. A pooled yield of rather more than 6 litres was filtered through terylene gauze and adjusted to $p \mathrm{H} \mathrm{6.5}$; sodium azide was added to $0.1 \%$. Finely particulate matter was separated by centrifugation. Simultaneous concentration and dialysis was carried out in a $37^{\circ} \mathrm{C}$ room, in the line of a continuously running fan, by the use of a glass funnel, $6 \frac{1}{2}$ feet of dialysis tubing, a 1 litre measuring cylinder, and a pump. The lower end of the dialysis tubing was immersed in N/4 buffer in the cylinder. Circulation was continually maintained within the dialysis system by a pump, and the system was enclosed to prevent inhalation of extract by persons using the room. Concentration and dialysis was continued for a week, with daily changes of buffer in the cylinder until a 25-30 fold reduction in volume had been effected. The concentrate was then cleared by final centrifugation, and is referred to as $N / 1$ antigen. Dilutions made in $0.85 \%$ saline are referred to as $N / 2, N / 16$ antigen, etc. Some of the N/1 antigen was further concentrated approximately two and a half times by use of carbowax (see under test sera) and is referred to as $2.5 \mathrm{~N}$ antigen.

The daily dialysates were saved and pooled. After adjustment of the $p \mathrm{H}$ of pooled dialysates to 6.5 and centrifugation, the supernatant was concentrated by evaporation at $37^{\circ} \mathrm{C}$ to approximately $80 \mathrm{ml}$. After $48 \mathrm{hr}$ at $4^{\circ} \mathrm{C}$, the concentrated dialysate was cleared, while still cold, by a short centrifugation. The resulting supernatant is subsequently referred to as dialysate.

AGAR-COATED SLIDES AND SLOT CUTTING One per cent Noble agar (Difco) was used throughout. Glass slides 3 in. $\times 1$ in., chemically cleaned and serially numbered with waterproof ink, were spread with $1 \%$ molten agar in deionized water. When set the slides were dried in the incubator, leaving an even coating of agar which prevented the numbers from being washed off. To coated slides on a level platform a unit volume of molten $1 \%$ agar in $\mathrm{N} / 2.5$ buffer was added by use of a marked Pasteur pipette delivering $2.25 \mathrm{ml}$. When set, six incisions were made simultaneously in the agar with a cutter. These enabled the removal of two $\frac{1}{8} \mathrm{in}$. slots or of three $\frac{1}{16}$ in. slots.

Prepared slides were laid on a marked card for transverse loading with antigen-impregnated strips at predetermined separations of $\frac{1}{2}$ in. or $\frac{3}{4}$ in. (see discussion). Except where otherwise mentioned, $\frac{1}{16}$ in. slots were used.

OSMOPHORESIS Osmophoresis was normally carried out overnight (15-24 hr) on slides set up as shown in Fig. 1, with a constant-voltage power pack setting between 50 and 80 volts and a current consumption between $1 \mathrm{ma}$ and 2.5 ma per slide. The variation in current was subsequently ascertained to be partly due to non-standardiza- tion in the use of the wicks, which were of Whatman 3 MM paper (see discussion).

WASHING AND STAINING On completion of osmophoresis, strips were removed by rinsing with running water. After the slots had been filled with molten agar, the slides were transferred to beakers containing $0.85 \%$ saline buffered

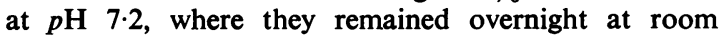
temperature. Drying and staining were as described by Pepys and Jenkins (1965) except that the slides were differentiated in stain solvent (without dye) containing $5 \%$ glycerol. Permanently non-brittle preparations resulted.

TEST SERA Forty-six sera were separated into five groups. Group A comprised 10 sera from typical cases of farmer's lung, all of which were positive by conventional immunodiffusion. The sera in all the remaining groups were negative by the conventional test. Group B comprised 16 sera from typical cases of farmer's lung; group C, four sera from suspected, clinically atypical, cases, group D, 12 sera from healthy farmers, and group E, four sera from urban asthmatics.

The four sera in group C (Table II) were from within the area served by this laboratory. The remainder were received from other laboratories within the Working Party. Sera for some of the tests were concentrated in small Perspex chambers, separated from a larger chamber by dialysis membrane. The lower ends of the small chambers were 'blind'. Dilute $p \mathrm{H} \mathbf{7 \cdot 2}$ buffer and carbowax were added to the larger central chamber, and concentration took place overnight in the refrigerator. The concentration factor was between 2.5 and 3 . All of the test sera except C.3 (in group A) and the four sera in group C, had been kept in storage for several years.

\section{RESULTS}

Results are set out in Tables I and II. Nine out of 10 sera from cases of farmer's lung which were positive by conventional immunodiffusion showed seven or more arcs of precipitation; the tenth showed four arcs. Except in serum LD, not more than two arcs were shown in nine out of 16 unconcentrated sera from typical cases of farmer's lung which were sero-negative by conventional immunodiffusion, in four out of 12 healthy farmers, in one out of four urban asthmatics, and in four out of four suspected cases of farmer's lung whose symptoms were not typical. Concentration of the sera resulted in 11 positives out of 12 sera tested in the 'sero-negative' group of typical cases, but did not substantially increase the proportion of positives in the other groups. Three or more arcs of precipitation were not shown by any sera, concentrated or unconcentrated, other than from typical cases of farmer's lung.

Tests with dialysate gave positive results with all the 'conventionally' sero-positive sera (except one which was not tested with dialysate), with two 'con- 
${ }^{1}$ For explanations of symbols see footnotes to Table II.

TABLE I

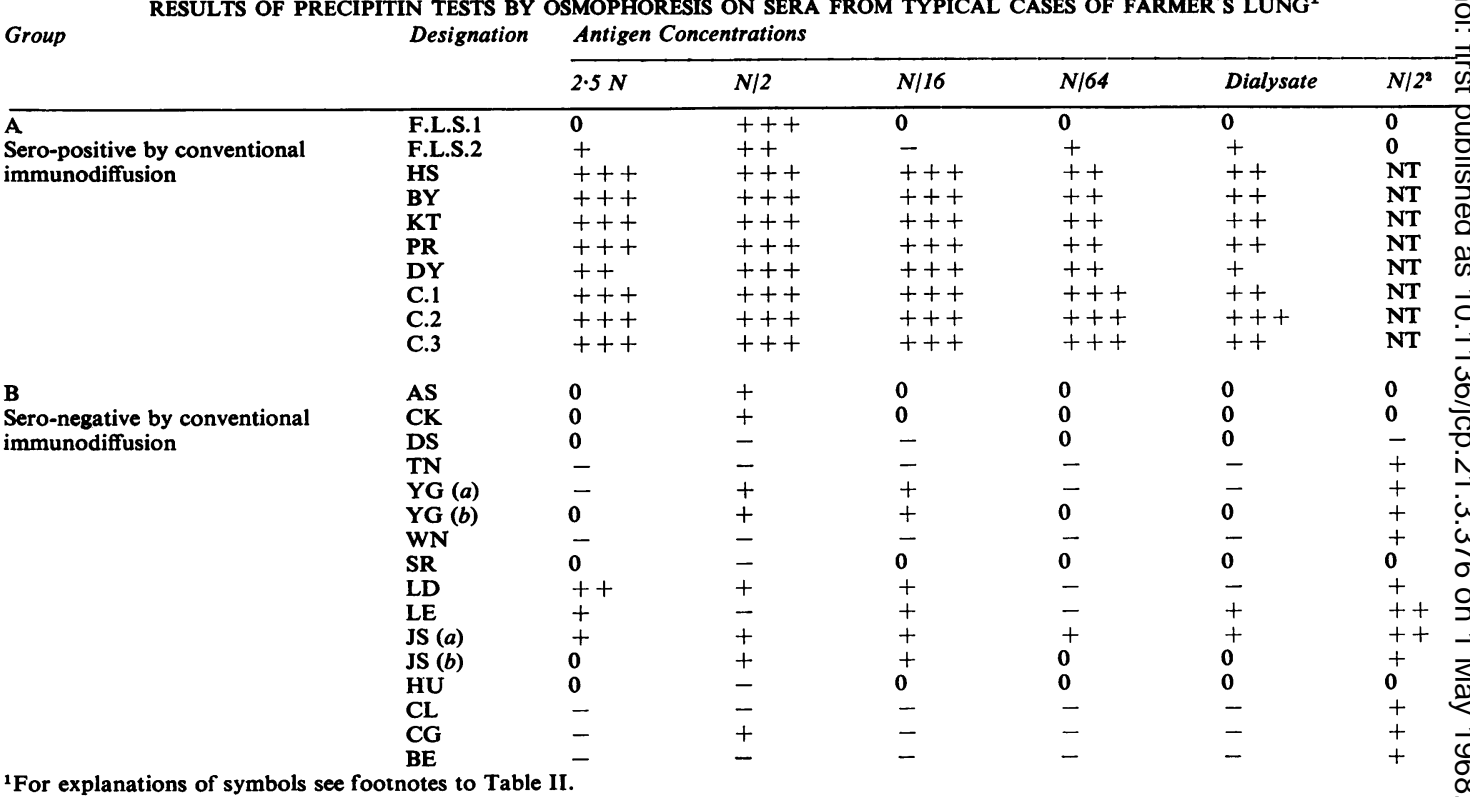

TABLE II

RESULTS OF PRECIPITIN TESTS, BY OSMOPHORESIS, ON CATEGORIES OF SERA OTHER THAN FROM TYPICAL CASES OF FARMER'S LUNG, NOT REACTING IN THE CONVENTIONAL DOUBLE DIFFUSION TEST

\begin{tabular}{|c|c|c|c|c|c|c|c|}
\hline \multirow[t]{2}{*}{ Group } & \multirow{2}{*}{$\begin{array}{l}\text { Designa- } \\
\text { tion }\end{array}$} & \multicolumn{6}{|c|}{ Antigen Concentrations } \\
\hline & & $2 \cdot 5 N$ & $N / 2$ & $N / 16$ & $N / 64$ & $\begin{array}{l}\text { Di- } \\
\text { alysate }\end{array}$ & $N / 2^{2}$ \\
\hline $\begin{array}{l}\text { C } \\
\text { Suspected } \\
\text { farmer's lung }\end{array}$ & $\begin{array}{l}\text { RE } \\
\text { NN } \\
\text { HL } \\
\text { TN }\end{array}$ & $\begin{array}{l}+ \\
+ \\
+ \\
+\end{array}$ & $\begin{array}{l}+ \\
\pm \\
+ \\
+\end{array}$ & $\begin{array}{l}+ \\
- \\
+\end{array}$ & $\begin{array}{l}+ \\
+ \\
+\end{array}$ & $\begin{array}{l}- \\
- \\
+\end{array}$ & $\begin{array}{l}+ \\
+ \\
+ \\
+\end{array}$ \\
\hline $\begin{array}{l}\text { D } \\
\text { Farmers } \\
\text { free of } \\
\text { symptoms } \\
\text { ie, healthy } \\
\text { men }\end{array}$ & $\begin{array}{l}\text { LS }^{1} \\
\text { MN } \\
\text { MC } \\
\text { OR } \\
\text { PT } \\
\text { CK } \\
\text { GM } \\
\text { LE } \\
\text { GT } \\
\text { ME } \\
\text { MY } \\
\text { LW }\end{array}$ & $\begin{array}{l}- \\
- \\
- \\
\overline{-} \\
- \\
- \\
\overline{-} \\
\overline{-} \\
+\end{array}$ & $\begin{array}{l}- \\
- \\
\overline{-} \\
+ \\
+ \\
\overline{-} \\
\overline{+} \\
- \\
\overline{+}\end{array}$ & $\begin{array}{l}\overline{-} \\
\overline{0} \\
\overline{+} \\
- \\
- \\
- \\
\overline{-} \\
\overline{-} \\
+\end{array}$ & $\begin{array}{l}- \\
- \\
\overline{-} \\
\overline{0} \\
\overline{ \pm} \\
\pm \\
\overline{-} \\
\overline{-} \\
\overline{0}\end{array}$ & $\begin{array}{l}- \\
- \\
- \\
0 \\
- \\
- \\
- \\
- \\
- \\
-\end{array}$ & $\begin{array}{l}- \\
- \\
- \\
\overline{+} \\
+ \\
\bar{t} \\
\pm \\
+ \\
- \\
+\end{array}$ \\
\hline $\begin{array}{l}\text { E } \\
\text { Urban } \\
\text { asthmatics }\end{array}$ & $\begin{array}{l}\text { DN } \\
\text { CM } \\
\text { MH } \\
\text { BS }\end{array}$ & $\begin{array}{l}- \\
- \\
-\end{array}$ & $\begin{array}{l}- \\
\overline{-} \\
\pm\end{array}$ & $\begin{array}{l}\overline{0} \\
\overline{+}\end{array}$ & $\begin{array}{l}\overline{0} \\
- \\
-\end{array}$ & $\begin{array}{l}- \\
- \\
-\end{array}$ & $\begin{array}{l}+ \\
- \\
\pm \\
\pm\end{array}$ \\
\hline
\end{tabular}

${ }^{1}$ This serum, when tested in the fresh state, reacted in the conventional double diffusion test.

'Tests in this column were with concentrated sera.

NT Not tested.

$O$ Insufficient serum for testing.

+++7 or more arcs of precipitation.

++ 3-6 arcs of precipitation.

$+1-2$ arcs of precipitation.

\pm Doubtful.

- No arcs of precipitation. ventionally' sero-negative sera from typical cases of farmer's lung, and with one suspected case. Nos reactions were given by dialysate with sera eithero from healthy farmers or from urban asthmatics.

\section{DISCUSSION}

Pepys et al. (1965), by conventional double diffusion techniques, found 164 reactors to $T$. polyspora? extract among 194 sera from farmers with farmer's lung and 25 reactors among 104 sera from farmers: without farmer's lung. The latter group included 3 . $47 \%$ of asthmatics amongst whom the incidence of reactors was nearly twice that in the healthy farmers. No reactors to $T$. polyspora extract were found in 1240 urban dwellers. Though the report does not make it clear how many sera which had not reacted witho mouldy hay extract in previous tests (Pepys, Riddell, Citron, and Clayton, 1962) were included within thiso latter series, no conscious bias was exercised in then selection of these sera (Pepys, personal communica-N tion, 1967).

Whatever the incidence may be, in healthy, and in asthmatic sera, of precipitins to $T$. polyspora ande moulds, the test now described seems likely to reveafs a fuller picture of that incidence than conventiona $L_{-}$ immunodiffusion. Caution should therefore be exercised in the interpretation of sera which showd less than three or four arcs of precipitation. Stronger reactions, and reactions with dialysate, seemed ine this limited series to be reliable indicators of farmer'se 


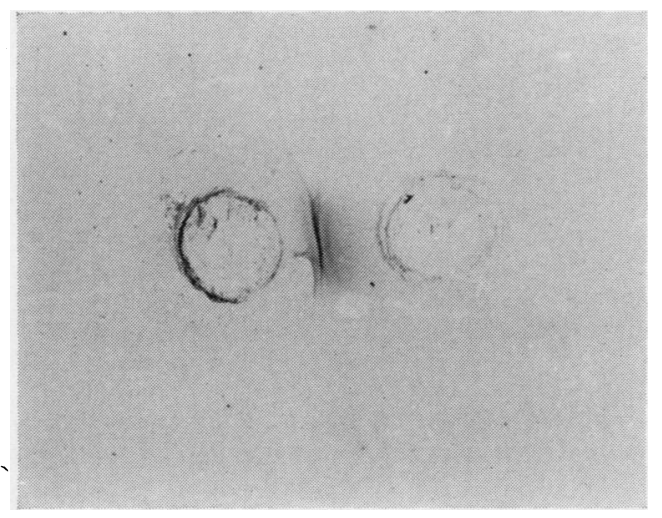

a Serum C.2, diluted 1/4 with non-reacting serum, in well on left, N/16 antigen in well on right; separation $5 \mathrm{~mm}$. Overnight osmophoresis at 60 volts.

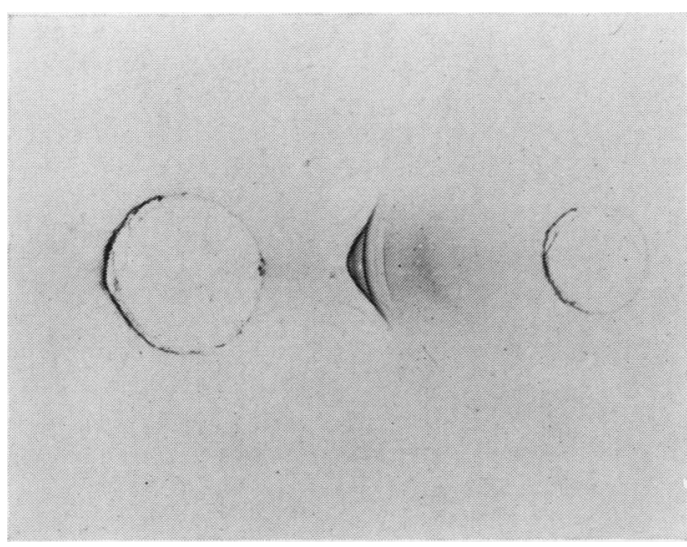

b Same as a except serum origin is larger and separation is $\frac{1}{2}$ in.

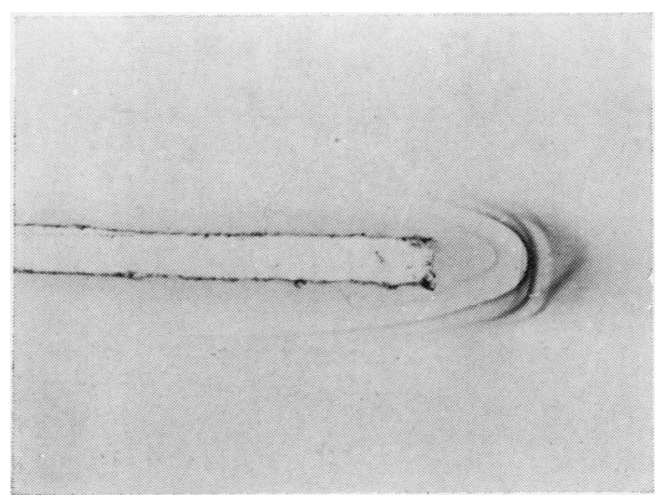

c Same as b except that antigen origin is a $\frac{1}{2}$ in. $\times$ $\frac{1}{16}$ in. transverse slot, and serum origin is a 1 in. $\times \frac{1}{16}$ in. longitudinal slot.

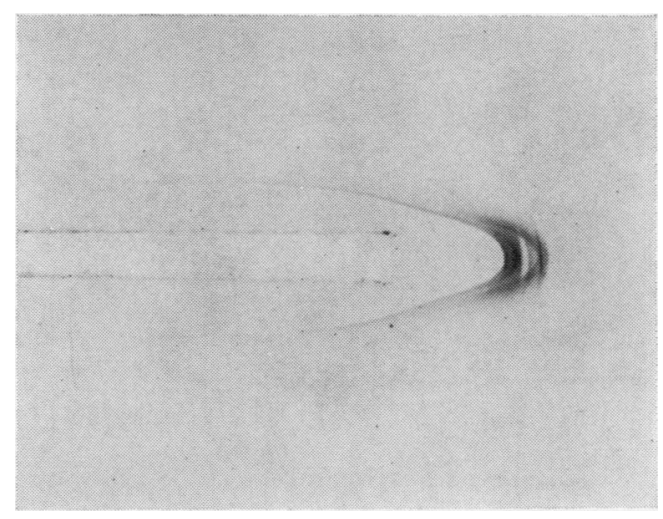

$\mathrm{d}$ Same as c except that antigen origin is in paper strip charged with $N / 2$ antigen.

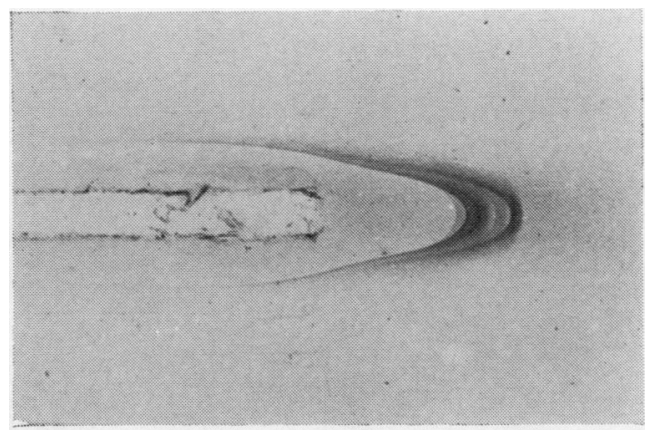

e Same as d except that separation is increased to $\frac{3}{4}$ in.

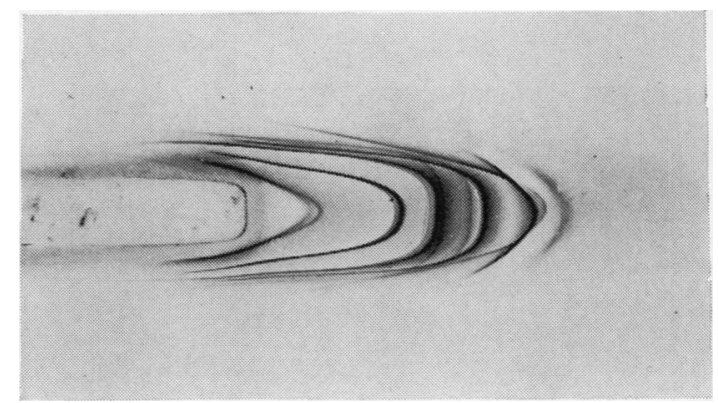

f Same serum (undiluted) in $\frac{1}{8}$ in. slot, and stronger antigen of a different batch. Wide separation ( $1 \frac{3}{8} \mathrm{in}$.) was used to obtain high resolution.

FIG. 2. Results with different sera using different antigens and separations for immunoosmophoresis. 


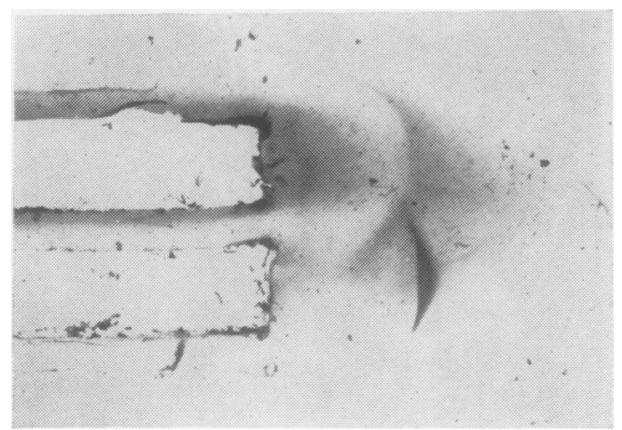

g Two $\frac{1}{8}$ in. serum slots. Upper, serum LD (concentrated): lower, serum $W$. Note crossing of the two arcs. (Concentration of $L D$ serum evidently adversely affected the equivalence with $2.5 \mathrm{~N}$ antigen obtained with $L D$ serum unconcentrated. In the latter case $L D$ serum showed three arcs of precipitation.)

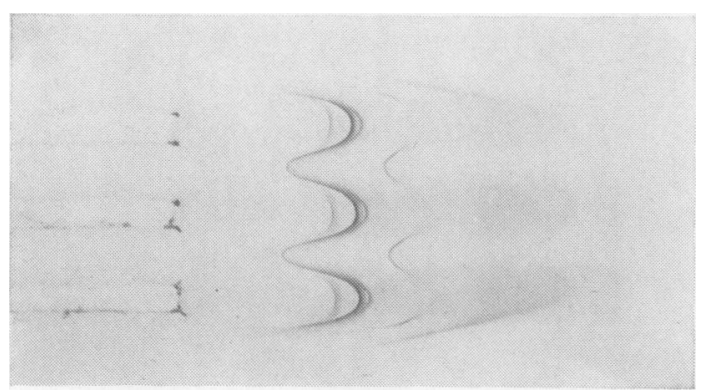

h A single positive serum added to all three slots. Note the 'reactions of identity' contrasting with non-identity in Figure $2 g$.

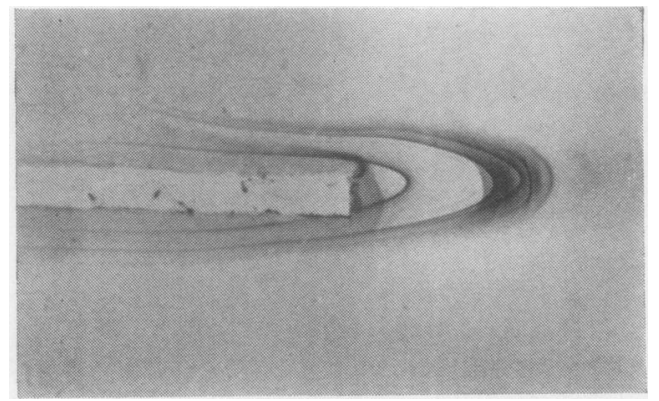

i Serum as in a-e. Separation $\frac{3}{4}$ in. Antigen of a different batch equivalent in strength to N/2. Osmophoresis in refrigerator for $48 \mathrm{hr}$ at 50 volts, no saline wash. Note acute bend on arc nearest to serum origin. A small degree of non-specific precipitin can also be seen. j Different serum and with no antigen present. Not non-specific precipitin around serum origin, after osmo phoresis.

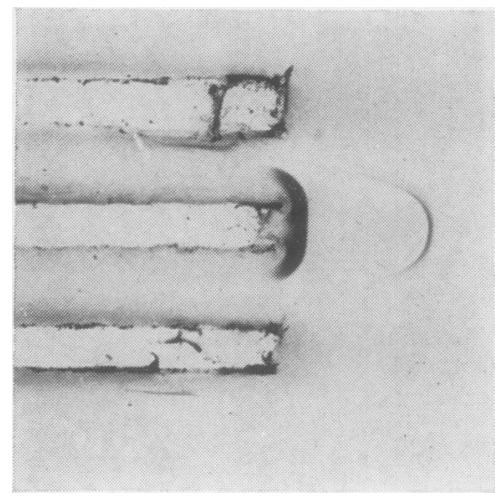

k Upper slot, WN; middle, JS (a); lower, YG (a). Notकृ non-specific precipitin opposite JS (a), as well as two. specific arcs, one of which, due to $B$ antigen, is bareli visible. One arc can also be seen opposite YG.

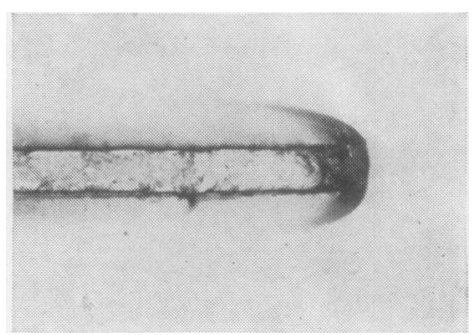

1 A further illustration of non-specific precipitin. 


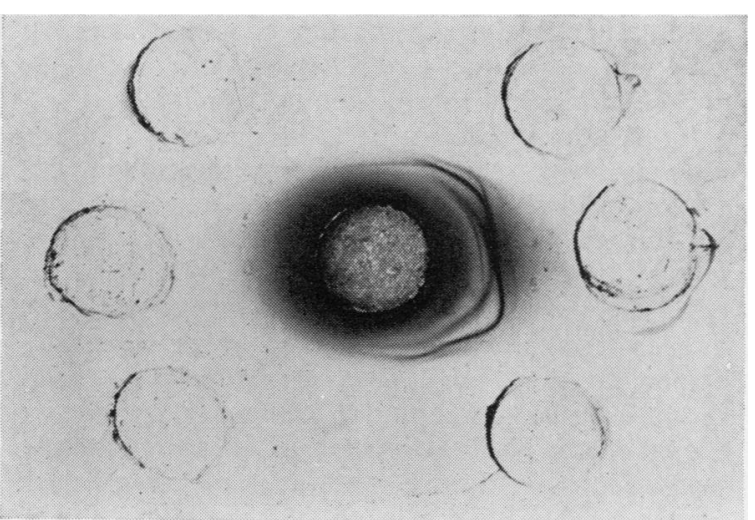

$\mathrm{m}$ An almost conventional immunodiffusion test in Tris-saline immunodiffusion buffer at $\mathrm{pH} 7 \cdot 4$. Central well contained serum as in a-e etc.; the six outer wells contained N/16 antigen. Osmophoresis was carried out in the usual way except that a 9 volt dry battery was substituted for the power pack. Note absence of arcs on the anodic side (left) of the serum well.

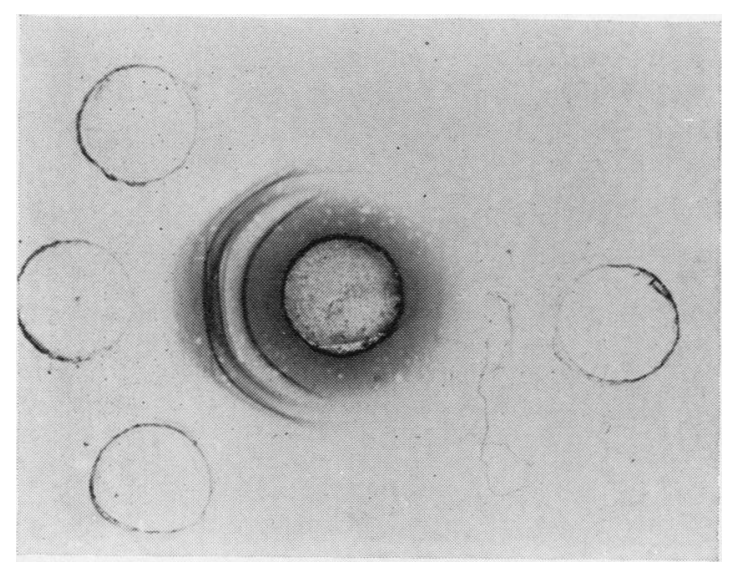

n Another example of overnight osmophoresis similar to 1, with the use of a 9 volt dry battery.

lung. Whether by chance or otherwise, the test with dialysate picked out the one patient in group $\mathrm{C}$ who had acute symptoms. A fully negative result, by the methods described here, would seem to have greater significance than a similar result by the conventional test.

The discovery, in the dialysate, of haptens capable of reacting with immune sera, was unexpected. It was at first supposed that there had been leakages during dialysis, through which 'normal' antigens had penetrated. This supposition was not supported by the selective nature of the reactions of the dialysate.
Further investigation is indicated, not only of dialysates, but also of 'normal' antigens of $T$. polyspora, with a view to differentiating the various types of antibody which may be found in various categories of sera. Only limited progress in this direction is likely with the sole use of multivalent antigens. It was ascertained for example that antibody in serum LD (group B) differed from that in serum LW (group D) (Fig. 2g). Serum LD was unusual in another respect. The strongest reaction given by this serum was with $2.5 \mathrm{~N}$ antigen. Less cumbersome methods of qualitative differentiation between sera should follow adequate fractionation of the polyvalent antigen. This has been achieved with varying degrees of success by all of the following methods: differential precipitation with alcohol, acetone, ammonium sulphate, and trichloracetic acid, filtration through Sephadex G150 and G100, and electrophoresis. A combination of two or more of these or other methods might be necessary for the satisfactory separation of single fractions.

Though osmophoresis, applied in this way, provided a sensitive test for precipitins, sensitivity probably was not the only factor which contributed to the finding of reactors in group B. It seems likely that the use of more highly concentrated antigens than are necessary for the detection of group A sera also contributed. While group A sera reacted adequately with $\mathrm{N} / 64$ antigen, more concentrated antigens were necessary in all but one of the group B sera, and $2 \cdot 5 \mathrm{~N}$ antigen, 160 times more concentrated, was necessary for the detection of two out of three precipitins found in serum LD. Group B sera may contain precipitins to antigens which are absent from, or present in insufficient concentration in, dilutions of purified polyvalent antigen currently used in conventional immunodiffusion tests. The latter are tailored according to the reactions they give with group A sera.

Discussion is necessary concerning practical aspects of the test, including interpretation. Some sera, especially during osmophoresis at a low temperature, show a type of precipitation which appears even when no antigen is present. This nonspecific precipitation must be distinguished from antigen-antibody combination. Non-specific precipitation can usually be recognized by the position and shape of the arc. It appears near to the serum origin, nearer than the arcs of the ' $B$ ' antigens (Pepys et al., 1963), which are acutely paraboloid in shape (Fig. 2i). Arcs of non-specific precipitation are bluntended and often show a 'Cupid's bow' effect, as though arcs had arisen separately from the two sides of the serum origin (Fig. $2 \mathrm{j}, \mathrm{k}, \mathrm{l}$ ). Confusion may be avoided by comparsion with a blank antigen control, or with a different antigen. 
Shapes of arcs due to antigen-antibody combination are determined by many factors which include the relative rates of migration of antigen and antibody, and lateral diffusion by antibody. With decreasing osmophoresis-potential the relative contribution made by lateral diffusion is increased. Since lateral diffusion of antibody results in its dilution, a high osmophoresis-potential might be advantageous. No doubt this holds true to a certain point, though too high a potential $(200 \mathrm{v}$.) resulted in blurring of the weaker arcs, with loss of sensitivity. This was presumably due to the reactants, migrating faster in opposite directions, having been in contact for insufficient time for full combination. An optimum osmophoresis-potential is difficult to determine and may differ with every pair of reactants as well as with their distances of initial separation. The presence of visible arcs in an unstained preparation is not necessarily an indication that osmophoresis has been brought to completion. Until the whole of the antibody present has combined with antigen, further osmophoresis may intensify arcs and increase their number. There is a wide latitude in osmophoresis-potential which may be applied (Fig. $2 \mathrm{~m}, \mathrm{n}$ ). This is fortunate owing to the many variables which influence that potential. The principles which underlie these variables are well known, but two which might be overlooked are worthy of mention. These are the dimensions (electrical conductivity) of the unsubmerged portions of the paper wicks, and the depth and ionicity (conductivity) of the tank buffer. Sustained osmophoresis results in precipitation of barbituric acid on the anode, with loss of buffer ionicity. Standardization in the use of paper wicks and frequent changes in tank buffer are therefore necessary in order to maintain constant conditions.

Further investigation is called for regarding the disappearance, during a wash in unbuffered saline at $37^{\circ} \mathrm{C}$ on a preparation from serum AS, of arcs of precipitation. Saline washes at $37^{\circ} \mathrm{C}$ had been routinely used for a few months previously, but no similar phenomenon had been observed with sera in group A. Even with the later use of buffered saline at room temperature, it was noticed that arcs arising from sera, other than group A sera, were sometimesmore conspicuous before staining, than after.

In all tests shown in the table the serum origins were in 1 in. $\times \frac{1}{16}$ in. slots, separated from the antigen origin by a distance either of $\frac{3}{4}$ in. or of $\frac{1}{2}$ in $\frac{5}{\omega}$ An increase in the width of the serum slot, and a reduction in the distance of separation below $\frac{3}{4}$ in 0 both slightly increased the sensitivity of the testion Similar increases in sensitivity were generally $\overrightarrow{0}$ inapparent in tests with more strongly reacting sera $\overrightarrow{-}$ except in respect of certain ' $C$ ' antigens (Pepys et al. W 1963), where the advantage gained by a shortes separation may have been considerable. Insufficien separation (less than $\frac{1}{2}$ in.) of the reactants, on theother hand, results in less good resolution and greater difficulty in the discrimination of non-specific precipitation.

Oxoid Ionagar no. 2 was used in tests not men? tioned elsewhere. Differences were noted in the relative positions, in the two agars, of various arcso but no evidence was found to suggest that these differences would materially have influenced the results given in the tables, had Ionagar been used ${ }^{\infty}$ Differences observed were in general consistent with the hypothesis that endosmosis is less active ir lonagar than in Noble agar. This might be arb advantage in the detection of ' $C$ ' antibodies bsö osmophoresis.

I am grateful to Dr. I. G. Murray for constant encourage ment, for doing conventional tests on some of the sera? for cultures of $T$. polyspora, and for sera. I wish to thank Dr. J. Pepys and Mr. P. A. Jenkins for sera, and for. supplying results of immunodiffusion tests. I also wish to thank Drs. D. G. Davies, B. Moore, and P. J. Wormald for sending sera. I am indebted to Mr. C. J. Webb angु Mr. S. T. Higgins for the photographs.

\section{REFERENCES}

Culliford, B. J. (1964). Nature (Lond.), 201, 1092.

Pepys, J., and Jenkins, P. A. (1965). Thorax, 20, 21. , Festenstein, G. N., Gregory, P. H., Lacey, M. E., and Skinner, F. A. (1963). Lancet, 2, 607.
Riddell, R. W., Citron, K. M., and Clayton, Y. M. (1962) Thorax, 17, 366. 